Published in final edited form as:

J Cancer Surviv. 2018 June ; 12(3): 398-406. doi:10.1007/s11764-018-0679-7.

\title{
Evolution of Neurocognitive Function in Long-term Survivors of Childhood Acute Lymphoblastic Leukemia Treated with Chemotherapy Only
}

\author{
Wei Liu, $\mathrm{PhD}^{1,2}$, Yin Ting Cheung, $\mathrm{PhD}^{3}$, Heather M Conklin, $\mathrm{PhD}^{4}$, Lisa M Jacola, $\mathrm{PhD}^{4}$, \\ DeoKumar Srivastava, $\mathrm{PhD}^{1}$, Vikki G Nolan, $\mathrm{DSc}^{2}$, Hongmei Zhang, $\mathrm{PhD}^{2}$, James G Gurney, \\ $\mathrm{PhD}^{2}$, I-Chan Huang, $\mathrm{PhD}^{3}$, Leslie L Robison, $\mathrm{PhD}^{3}$, Ching-Hon Pui, MD ${ }^{5}$, Melissa $\mathbf{M}$ \\ Hudson, MD ${ }^{3,5}$, and Kevin R Krull, PhD $^{3,4}$ \\ ${ }^{1}$ Department of Biostatistics, St. Jude Children's Research Hospital \\ ${ }^{2}$ School of Public Health, University of Memphis \\ ${ }^{3}$ Department of Epidemiology and Cancer Control, St. Jude Children's Research Hospital \\ ${ }^{4}$ Department of Psychology, St. Jude Children's Research Hospital \\ ${ }^{5}$ Department of Oncology, St. Jude Children's Research Hospital
}

\section{Abstract}

Purpose-To determine the evolution of neurocognitive problems from therapy completion to long-term follow-up in survivors of childhood acute lymphoblastic leukemia treated with chemotherapy-only.

\begin{abstract}
Methods-We evaluated whether attention problems observed at therapy completion evolve into long-term executive dysfunction in 158 survivors treated on a single institution protocol.

Treatment data (high dose intravenous methotrexate exposure [serum concentration] and triple intrathecal chemotherapy injections) were collected. Parent-report of behavior and direct cognitive testing of survivors was conducted at end of therapy, and survivors completed neurocognitive testing when $>5$ years post-diagnosis.
\end{abstract}

\footnotetext{
Corresponding author: Kevin R. Krull, PhD. Department of Epidemiology and Cancer Control, St. Jude Children's Research Hospital, 262 Danny Thomas Place, MS 735, Memphis, TN 38105. kevin.krull@stjude.org; (v) 901-595-5891; (f) 901-595-5845.

Ethical approval: "All procedures performed in studies involving human participants were in accordance with the ethical standards of the institutional and/or national research committee and with the 1964 Helsinki declaration and its later amendments or comparable ethical standards."

Conflict of Interest

All authors have no conflict of interest including financial interests, activities, relationships, and affiliations; sources of funding and support.

Authors' contributions

The following authors have made substantial contributions to the intellectual content of the paper, including conception/design (WL, YTC, DS, KRK), acquisition, analysis, or interpretation of data (WL, YTC, HMC, LMJ, DS, VGN, HZ, JGG, IH, LLR, CHP, MMH, KRK), drafting of the manuscript (WL, YTC, KRK) and critical revision of the manuscript for important intellectual content (WL, YTC, HMC, LMJ, DS, VGN, HZ, JGG, IH, LLR, CHP, MMH, KRK). Contributions also include statistical analysis (WL, DS), obtaining funding (KRK, LLR, MMH). All authors have approved the final version of this manuscript. KRK had full access to all data in the study and had final responsibility for the decision to submit for publication.
} 
Results-At end of chemotherapy, survivors (52\% female; mean age 9.2 years) demonstrated higher frequency of impairment in sustained attention (38\%) and parent-reported inattention (20\%) compared to population expectations (10\%). At long-term follow-up, survivors (mean age 13.7 years; 7.6 years post-diagnosis) demonstrated higher impairment in executive function (flexibility $24 \%$, fluency $21 \%$ ), sustained attention (15\%), and processing speed (15\%). Sustained attention improved from end of therapy to long-term follow-up ( $\mathrm{p}<0.001)$. Higher methotrexate AUC and greater number of intrathecal injections were associated with attention problems $(\mathrm{p}=0.009, \mathrm{p}=0.002$, respectively) at the end of chemotherapy and executive function ( $\mathrm{p}<0.001$, $\mathrm{p}=0.02$, respectively) problems at long-term follow-up. Attention problems at the end of therapy were not associated with executive function problems at long-term follow-up (p's $>0.05$ ). The direct effect of chemotherapy exposure predicted outcomes at both time points.

Conclusions and Implications for Cancer Survivors-Survivors should be monitored for neurocognitive problems well into long-term survivorship, regardless of whether they show attention problems at the end of therapy. Treatment exposures are the best predictor of long-term complications.

\section{Keywords}

Childhood Acute Lymphoblastic Leukemia; Childhood cancer; Chemotherapy; Attention; Executive Function; Behavior

\section{Introduction}

Contemporary chemotherapy results in 5-year survival of greater than $90 \%$ for children with acute lymphoblastic leukemia (ALL) without the use of cranial radiation therapy (CRT) [1, 2]. Compared to survivors treated on older protocols that included CRT, neurocognitive outcomes for survivors treated with chemotherapy only have significantly improved, but problems are still identified at a higher frequency than that expected in the general population $[3,4]$.

Cognitive problems emerge early during the course of chemotherapy-only treatment for childhood ALL. Compared to normative data, ALL survivors demonstrate lower verbal and visual working memory, and slower motor speed after completion of induction therapy, which predict decline in visual-motor integration skills over time [5]. By the end of therapy, all survivors appear to be at risk for problems in sustained attention, and those treated on higher risk protocols are reported by parents to have more problems with inattention compared to survivors treated on low risk protocols $[6,7]$.

Neurocognitive problems in long-term survivors of childhood ALL are often concentrated in higher order cognitive abilities (i.e., executive function) [3]. Among young adult survivors of childhood ALL who were treated with chemotherapy-only and who were at least ten years from diagnosis, the frequency of severe impairment in executive function was eight times higher than that seen in the general population [4]. Although higher plasma concentration of methotrexate and homocysteine are associated with lower executive function in long-term adolescent survivors [8], some studies failed to identify treatment- or disease-related factors associated with executive function problems [9]. 
With typical development, executive functions emerge early in childhood and continue to strengthen throughout adolescence [10]. These executive functions begin with core skills in inhibitory control, attention and working memory [11], and evolve into the higher order abilities of cognitive fluency, flexibility, planning, organization and abstraction [12]. Impairment in core skills may limit development of the higher order abilities.

The aim of this study was to evaluate the association between sustained attention and parentrated behavioral inattention at the end of chemotherapy, with neurocognitive outcomes (i.e. executive function, sustained attention and processing speed) at long-term follow-up. We were interested in determining whether specific chemotherapeutic exposures independently predict neurocognitive impairment at both short-term and long-term follow-up, and whether short-term attention and behavioral problems evolve into long-term problems with executive function. If long-term neurocognitive outcomes are predicted by end of therapy symptoms, these early symptoms may serve as a marker for those at greatest risk and in most need of early intervention.

\section{Method}

\section{Patients}

This study was approved by the institutional review board at St. Jude Children's Research Hospital and informed consent was obtained from the parents and assent from the survivors, as appropriate.

From 2000 to 2010, 408 children with ALL were treated at St. Jude Children's Research Hospital on the Total Therapy XV protocol (ClinicalTrials.gov, NCT00137111).[2] For endof-therapy assessment, survivors had to be in continuous complete remission, not have a preexisting non-cancer related neurodevelopmental or genetic disorder associated with cognitive impairment, not have a brain injury unrelated to their cancer, and be proficient in English. There were a total of 262 survivors evaluated at the end of therapy. To be eligible for long-term follow-up assessment, survivors must have been more than five years from diagnosis and more than eight years of age (to reliably and uniformly assess executive function abilities), remained in continuous complete remission, and not experienced an unrelated brain injury after cessation of therapy. There were 220 survivors eligible for longterm follow-up assessment, 158 survivors participated (72\%; see Consort) and provided evaluable data.

Per the Total Therapy XV protocol, all children received high-dose intravenous methotrexate (HD-MTX), triple intrathecal treatments (methotrexate, hydrocortisone, cytarabine) and oral dexamethasone, in addition to other chemotherapeutic agents. Children in the low-risk and standard-risk arms were treated with four doses of HD-MTX at $2.5 \mathrm{gm} / \mathrm{m}^{2}$ and $5 \mathrm{gm} / \mathrm{m}^{2}$ per dose, respectively. Exposures to HD-MTX was measured as previously described,[13] Blood samples were drawn before HD-MTX infusion and at 6,23, and 42 hours following the start of each HD-MTX course. Exposures were quantified using area-under-the-curve (AUC) methods. Leucovorin rescue (10 to $15 \mathrm{mg} / \mathrm{m}^{2}$ ) started 42 hours after HD-MTX and repeated every six hours for a total of five doses. Prophylactic CRT was not administered to any 
patient, regardless of presenting features, including the presence of CNS leukemia at diagnosis.

\section{Neurocognitive Testing}

Sustained attention and parent-reported behavior problems were evaluated at a median of 120 weeks (range 113-130 weeks) post completion of consolidation (end of therapy) using standardized measures with demonstrated reliability and validity. Patients completed a computerized sustained attention measure (Conners' Continuous Performance Test CPT II) [14], which yields scores for omissions, commissions, variability, detectability, and a risk taking response style (i.e. speed-accuracy trade-off). Parents completed standardized ratings of child behavior (Conners' Parent Rating Scale CPRS) resulting in five scales, including conduct problems, inattention, somatic symptoms, anxiety, and hyperactivity/impulsivity [15].

At long-term follow-up, the Delis-Kaplan Executive Function System (DKEFS) [16] was used to assess cognitive flexibility (Number letter Switching), letter fluency, number sequencing, letter sequencing, planning (Tower), and problem solving abilities (Abstraction, 20 questions). The Wechsler Intelligence Scales $[17,18]$ were used to assess verbal working memory (Digit Backward), inhibitory control (Color-Word Interference Test), verbal span (Digit Forward) and spatial span (Spatial Forward), processing speed for visual (Symbol Search) and visual-motor (Digit Symbol). Rey Complex Figure Copy [19] was used to measure organization skills. The Grooved Pegboard Test was used as an index of motor speed, as it is a time-based measure that requires [20]. The same sustained attention measure included at end of therapy was administered at long-term follow-up [14].

\section{Statistical Analysis}

For demographic and clinical variables, means and standard deviations were calculated for continuous variables and two-sample t-tests were conducted to compare groups of participant and non-participants. Frequencies were provided for categorical variables and Chi-Square tests were used to assess significance. Behavioral and neurocognitive scores were transformed into age-adjusted Z-scores $(\mu=0, \sigma=1.0)$ using national normative data. Impairment of behavioral and neurocognitive outcomes were determined using a threshold of $10 \%$ of the normative sample, i.e. $\mathrm{z}$-score $\leq-1.286$ on survivor performances and $\mathrm{z}$-score $\geq 1.286$ for parent reports. One-sample t-tests were applied to test if mean survivor performances and parent reports were different from 0 , and one sample binomial tests were applied to compare percent of impairment with that of population norms." For comparisons with national norms from one-sample t-tests and binomial tests, adjustment for multiple hypothesis testing was conducted, and the FDR-controlling $p$-values with linear step-up method[21] were reported using MULTTEST procedure in SAS.

Among those end of therapy and long-term follow-up variables that were significantly different from population norms, for both mean scores and proportion impaired, general linear models (GLM) were used to identify associations with predefined pharmacological predictors [4, 8] (HD-MTX exposure, number of intrathecal injections), adjusting for age at diagnosis and parents' education. GLM was also used to test associations with risk 
classification, adjusting for parent education. Correlation analysis was applied to test associations between sustained attention and parent-rated behavior problems at the end of therapy with long-term neurocognitive outcomes. Degree of change in sustained attention zscore from end of therapy to long-term follow-up was tested using a one sample t-test, and the average changes were compared between males and females using two-sample t-tests. For any end of therapy outcomes that were significantly associated with pharmacological predictors and with long-term outcomes, mediation analysis was conducted to assess if the pharmacological effect on long-term outcomes was mediated by end of therapy function. All analyses were conducted in SAS (SAS 9.4, SAS Institute, Cary NC) or STATA 13.1 (StataCorp LP, College Station, TX).

\section{Results}

Characteristics of the eligible study population are presented in Table 1. There were no significant differences observed between participants and non-participants in demographics, risk stratum or cumulative drug doses. Of the 158 participants, $52 \%$ were females, $77 \%$ were White, and $43 \%$ were treated on the standard-risk arm.

At the end of therapy, sustained attention and parent-reported behavior problems were significantly worse than the population mean, with higher proportion of impairment than the $10 \%$ expected in omissions, variability, detectability, and risk taking, as well as parentreported attention problem, somatic symptoms and anxiety (all p-values $<0.05$ Table 2 ).

Long-term follow-up neurocognitive measures were significantly below the population mean, with higher proportion of impairment than the expected 10\%, on measures of executive function (abstraction, cognitive flexibility, letter fluency organization), attention (verbal span) and processing speed (letter sequencing, motor speed and visual-motor speed), with all p-values $<0.05$, Table 2 . However, compared to the frequency of impairment on sustained attention at the end of therapy, $52 \%$ survivors who demonstrated at least one sustained attention problems at the end of therapy did not show executive dysfunction at long-term follow-up, 23\% survivors demonstrated impairments at both time, $8 \%$ survivors displayed executive dysfunction from at least one measurement at long-term follow-up only, and $17 \%$ survivors did not display impairment at either time. Survivors improved on measures of sustained attention by an average of one to two standard deviations from end of therapy to long-term follow-up, and females demonstrated slightly more improvement than males (Figure 1).

Parent-reported inattention at the end of therapy was positively associated with HD-MTX exposure ( $\mathrm{p}=0.009)$ and number of IT injections ( $\mathrm{p}=0.002$, Online Resource 1$)$, and Standard $(\mathrm{p}=0.02$, Online Resource 3). HD-MTX exposure and number of IT injections were also associated with cognitive flexibility, omissions and visual-motor processing speed at longterm follow-up, with all p-values $<0.05$ (Online Resource 2). Standard risk was also associated with cognitive flexibility $(\mathrm{p}=0.02)$ and letter fluency $(\mathrm{p}=0.03$, Online Resource 3 ).

Sustained attention at the end of therapy predicted letter fluency, motor speed and visualmotor processing speed at long-term follow-up. Parent-reported inattention at the end of 
therapy was associated with verbal span, motor speed and visual-motor processing speed at long-term follow-up. Parent-reported hyperactivity/impulsivity at the end of therapy was associated with cognitive flexibility, organization, verbal span, motor speed and visual-motor processing speed at long-term follow-up (all p-values $<0.05$, Online Resource 4).

Based on mediation analysis (Table 3), only direct effects of treatment exposure were identified for measures of executive function and attention at long-term follow-up, suggesting that end of therapy performance and behavior did not mediate these outcomes (Figure 2a). The number of IT injections demonstrated a direct effect on processing speed at long-term follow-up $(\beta=-0.044, p=0.02)$, as well as an indirect effect through parentreported inattention $(\beta=-0.013, p=0.04$ Figure $2 b)$.

\section{Discussion}

We conducted a longitudinal study on the evolution of neurocognitive functions in a large sample of long-term survivors of childhood ALL treated on a contemporary chemotherapyonly regimen. Our novel results demonstrate that many survivors who have poor sustained attention at the end of therapy significantly improved with long-term follow-up. While most survivors were functioning well at long-term follow-up, a higher frequency of executive dysfunction and slow processing speed was identified compared to the general population. Parent-reported inattention at end of therapy and direct assessment of executive dysfunction and slow processing speed at long-term follow-up were associated with higher methotrexate exposure and a greater number of intrathecal chemotherapy treatments. Parent-reported inattention may also be due, in part, to parent's awareness of the treatments their child received. However, the executive function problems at long-term follow-up were not dependent upon development of inattention at the end of therapy. These findings suggest that patients who are going to receive higher concentrations of intravenous methotrexate and more intrathecal treatments will be at greater risk for long-term neurocognitive deficits, and thus should receive early therapeutic interventions during or shortly after active chemotherapy treatment.

Executive function begins with development of self-regulation and inhibitory control during early childhood [11, 22]. Self-regulation facilitates the development of attention and behavioral control [23, 24]. With maturation, higher order executive functions like cognitive flexibility and fluency develop $[11,25]$. Impairment in basic skills like attention may limit the development of these higher order functions. For this reason, we expected inattention at the end of therapy to impact the development of executive function at long-term follow-up. In the current study, many survivors demonstrated poor sustained attention at the end of therapy, though significant improvements in these attention skills were observed with longterm follow-up. Patients continued to receive 6MP and oral MTX up to 120 weeks. Those patients who were tested while still on the last weeks of maintenance therapy may have experienced acute effects of this therapy, while those patients tested after the end of maintenance therapy may have experienced lingering effects of the preceding treatments. Many survivors also demonstrated executive function problems during long-term follow-up, though these problems were not dependent upon the earlier attention problems. The sustained attention problems for some survivors identified by direct assessment at the end of 
therapy could be due to acute reactions to treatment that recover over time and do not impact executive function at long-term follow-up. We have previously shown that some survivors demonstrate recovery of cognitive problems from the beginning to the end of chemotherapy for childhood ALL [5].

At long-term follow-up, we found executive function problems to be much more prevalent than attention problems. Previous research has shown that prefrontal cortex regions are susceptible to HD-MTX exposure [8]. These regions are involved in executive function processes $[26,11]$ and mature over a longer period of time compared to many other brain regions [27]. White matter volume in this region and leukoencephalopathy in this region have been demonstrated to occur during chemotherapy and to be related to cognitive problems [28]. This may suggest that the chemotherapy impact on frontal lobe brain development may not be expressed through neurocognitive problems until long-term followup.

The current study demonstrated an association between the number of triple intrathecal injections and long-term processing speed was partially mediated through parent-reported inattention behavior at the end of therapy. Higher scores on parent-reported inattention indicate that parents perceive their children as having more problems "organizing their work", more difficulty "completing tasks or schoolwork", and more trouble "concentrating on tasks that require sustained mental effort" [15]. Many of these behaviors may be impacted by not only inattention, but also processing speed. For example, sustained mental effort will require longer duration with slower processing speed, and schoolwork is more likely to be incomplete when processing speed is slow. Thus, in rating their child's inattention symptoms at the end of therapy, parents may be picking up on subtle difficulties with processing speed as well. Parent-reported inattention behavior may be useful as an early marker for long-term problems in processing speed and task completion.

Several limitations should be considered in the current study. There was no healthy control group. However, we have a relatively large sample of childhood ALL survivors, permitting within group comparison across treatment exposures. In addition, the primary focus of the study was to determine how early attention problems evolve over time and, given the longitudinal follow-up, tracking of survivor change from baseline was possible. The sustained attention measure used in this study (CPT II) was normed on a sample that was $47 \%$ White, which may influence the frequency of identified impairment in our sample (70\% White). However, our difference in demographics would be included at both baseline and follow-up time points and, thus, should not account for the change in attention performance over time. An additional limitation is that there was no pre-therapy testing of neurocognitive functions, which is difficult to obtain in young children with this diagnosis. To account for this limitation we adjusted analyses for parent education, which should provide a reasonable surrogate for pre-morbid potential [29]. A final limitation was that we conducted a single site study, potentially limiting generalization. However, the chemotherapy agents used in our protocol comprise the backbone of contemporary protocols at most institutions. 
In conclusion, the current study demonstrates an association between chemotherapy exposure and short-term and long-term neurocognitive functions. Although short-term attention problems are correlated with long-term neurocognitive outcomes, the attention problems do not appear to evolve into executive function problems. Rather, both problems are driven by intensity of chemotherapy treatment. Future prospective studies should consider preventative intervention, particularly for those survivors who are going to be treated with more intensive chemotherapy regimens. In addition, survivors of childhood ALL should be closely monitored for neurocognitive problems well into long-term survivorship, regardless of whether they display problems at the end of chemotherapy.

\section{Supplementary Material}

Refer to Web version on PubMed Central for supplementary material.

\section{Acknowledgments}

Funding: This study was funded by NIMH (R01-MH085849 to KR Krull), NCI (U01-CA195547 to MM Hudson/LL Robison), and American Lebanese Syrian Associated Charities.

We thank Dr. Mary Relling, PharmD, St. Jude Children's Research Hospital and her lab to provide pharmacokinetic data. Pia Banerjee, PhD, Joycelynn Butler, MS, William Lewis, MS, Adrienne Studaway, Med, Adrienne Studaway, Med, Cynthia Jones, MA, Deborah Stewart, MA, all from Cognitive Neuroscience group, St. Jude Children's Research Hospital for providing technical support.

\section{References}

1. Iyer NS, Balsamo LM, Bracken MB, Kadan-Lottick NS. Chemotherapy-only treatment effects on long-term neurocognitive functioning in childhood ALL survivors: a review and meta-analysis. Blood. 2015; 126(3):346-53. DOI: 10.1182/blood-2015-02-627414 [PubMed: 26048910]

2. Pui CH, Campana D, Pei D, Bowman WP, Sandlund JT, Kaste SC, et al. Treating childhood acute lymphoblastic leukemia without cranial irradiation. The New England journal of medicine. 2009; 360(26):2730-41. DOI: 10.1056/NEJMoa0900386 [PubMed: 19553647]

3. Cheung YT, Krull KR. Neurocognitive outcomes in long-term survivors of childhood acute lymphoblastic leukemia treated on contemporary treatment protocols: A systematic review. Neuroscience and biobehavioral reviews. 2015; 53:108-20. DOI: 10.1016/j.neubiorev.2015.03.016 [PubMed: 25857254]

4. Krull KR, Brinkman TM, Li C, Armstrong GT, Ness KK, Srivastava DK, et al. Neurocognitive outcomes decades after treatment for childhood acute lymphoblastic leukemia: a report from the $\mathrm{St}$ Jude lifetime cohort study. Journal of clinical oncology: official journal of the American Society of Clinical Oncology. 2013; 31(35):4407-15. DOI: 10.1200/jco.2012.48.2315 [PubMed: 24190124]

5. Krull KR, Hockenberry MJ, Miketova P, Carey M, Moore IM. Chemotherapy-related changes in central nervous system phospholipids and neurocognitive function in childhood acute lymphoblastic leukemia. Leukemia \& lymphoma. 2013; 54(3):535-40. DOI: 10.3109/10428194.2012.717080 [PubMed: 22856670]

6. Conklin HM, Krull KR, Reddick WE, Pei D, Cheng C, Pui CH. Cognitive outcomes following contemporary treatment without cranial irradiation for childhood acute lymphoblastic leukemia. Journal of the National Cancer Institute. 2012; 104(18):1386-95. DOI: 10.1093/jnci/djs344 [PubMed: 22927505]

7. Jacola LM, Krull KR, Pui CH, Pei D, Cheng C, Reddick WE, et al. Longitudinal Assessment of Neurocognitive Outcomes in Survivors of Childhood Acute Lymphoblastic Leukemia Treated on a Contemporary Chemotherapy Protocol. Journal of clinical oncology: official journal of the American Society of Clinical Oncology. 2016; 34(11):1239-47. DOI: 10.1200/jco.2015.64.3205 [PubMed: 26858334] 
8. Krull KR, Cheung YT, Liu W, Fellah S, Reddick WE, Brinkman TM, et al. Chemotherapy Pharmacodynamics and Neuroimaging and Neurocognitive Outcomes in Long-Term Survivors of Childhood Acute Lymphoblastic Leukemia. Journal of clinical oncology: official journal of the American Society of Clinical Oncology. 2016; 34(22):2644-53. DOI: 10.1200/jco.2015.65.4574 [PubMed: 27269941]

9. Kanellopoulos A, Andersson S, Zeller B, Tamnes CK, Fjell AM, Walhovd KB, et al. Neurocognitive Outcome in Very Long-Term Survivors of Childhood Acute Lymphoblastic Leukemia After Treatment with Chemotherapy Only. Pediatric blood \& cancer. 2016; 63(1):133-8. DOI: 10.1002/ pbc.25690 [PubMed: 26312396]

10. Anderson P. Assessment and development of executive function (EF) during childhood. Child neuropsychology: a journal on normal and abnormal development in childhood and adolescence. 2002; 8(2):71-82. DOI: 10.1076/chin.8.2.71.8724 [PubMed: 12638061]

11. Diamond A. Executive functions. Annual review of psychology. 2013; 64:135-68. DOI: 10.1146/ annurev-psych-113011-143750

12. Romine CB, Reynolds CR. A model of the development of frontal lobe functioning: findings from a meta-analysis. Applied neuropsychology. 2005; 12(4):190-201. DOI: 10.1207/ s15324826an1204_2 [PubMed: 16422660]

13. Bhojwani D, Sabin ND, Pei D, Yang JJ, Khan RB, Panetta JC, et al. Methotrexate-induced neurotoxicity and leukoencephalopathy in childhood acute lymphoblastic leukemia. Journal of clinical oncology: official journal of the American Society of Clinical Oncology. 2014; 32(9):94959. DOI: 10.1200/jco.2013.53.0808 [PubMed: 24550419]

14. Conners, CKSM. Conners' Continuous Performance Test II (CPT II V. 5). North Tonawanda, NY: Multi-Health Systems Inc; 2000.

15. Conners, C. Conners Rating Scales-Revised: Technical Manual. New York: Multi-Health Systems. (Inc); 1997.

16. Delis, DC., Kaplan, E., Kramer, JH. Delis-Kaplan Executive Function System. San Antonio, TX: Psychological Corporation; 2001.

17. Wechsler, D. Wechsler Intelligence Scale for Children. 4. Harcourt Assessment, Inc; San Antonio, TX: 2003.

18. Wechsler, D. Wechsler Adult Intelligence Scale. 4. San Antonio: Pearson; 2008.

19. Meyers, JEMKR. Rey Complex Figure Test and Recognition Trial: Professional manual. Odessa, FL: Psychological Assessment Resources; 1995.

20. Lafayette Instrument Grooved Pegboard Test User Instructions. Lafayett IN: Lafatette Instrument@; 2002.

21. Benjamini Y, Hochberg Y. Controlling the False Discovery Rate: A Practical and Powerful Approach to Multiple Testing. Journal of the Royal Statistical Society Series B (Methodological). 1995; 57(1):289-300.

22. Best JR, Miller PH. A Developmental Perspective on Executive Function. Child development. 2010; 81(6):1641-60. DOI: 10.1111/j.1467-8624.2010.01499.x [PubMed: 21077853]

23. Barkley RA. Behavioral inhibition, sustained attention, and executive functions: constructing a unifying theory of ADHD. Psychological bulletin. 1997; 121(1):65-94. [PubMed: 9000892]

24. Ganesalingam K, Sanson A, Anderson V, Yeates KO. Self-regulation as a mediator of the effects of childhood traumatic brain injury on social and behavioral functioning. Journal of the International Neuropsychological Society: JINS. 2007; 13(2):298-311. DOI: 10.1017/s1355617707070324 [PubMed: 17286887]

25. Klenberg L, Korkman M, Lahti-Nuuttila P. Differential development of attention and executive functions in 3- to 12-year-old Finnish children. Developmental neuropsychology. 2001; 20(1):40728. DOI: 10.1207/s15326942dn2001_6 [PubMed: 11827096]

26. Duncan J, Owen AM. Common regions of the human frontal lobe recruited by diverse cognitive demands. Trends in neurosciences. 2000; 23(10):475-83. [PubMed: 11006464]

27. Blakemore SJ, Choudhury S. Development of the adolescent brain: implications for executive function and social cognition. Journal of child psychology and psychiatry, and allied disciplines. 2006; 47(3-4):296-312. DOI: 10.1111/j.1469-7610.2006.01611.x 
28. Reddick WE, Taghipour DJ, Glass JO, Ashford J, Xiong X, Wu S, et al. Prognostic factors that increase the risk for reduced white matter volumes and deficits in attention and learning for survivors of childhood cancers. Pediatric blood \& cancer. 2014; 61(6):1074-9. DOI: 10.1002/pbc. 24947 [PubMed: 24464947]

29. Noble KG, Houston SM, Brito NH, Bartsch H, Kan E, Kuperman JM, et al. Family income, parental education and brain structure in children and adolescents. Nature neuroscience. 2015; 18(5):773-8. DOI: 10.1038/nn.3983 [PubMed: 25821911] 


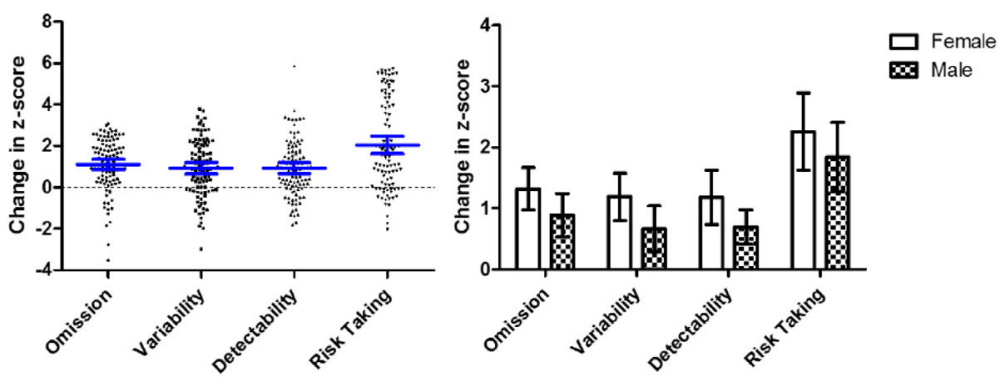

Figure 1.

On the left, change in sustained attention z-score from end of therapy to long-term followup. Each individual survivor is plotted in the figure, with scores above zero representing improvement and scores below zero representing decline. Survivors improved by an average of 1 to 2 standard deviations from end of therapy to long-term follow-up. The blue lines and error bars showed the mean and $95 \% \mathrm{CL}$ of z-score changes. On the right, mean and $95 \%$ CL for change in sustained attention from end of therapy to long-term follow-up stratified by sex. Females demonstrated slightly more improvement than males. 
Figure 2a

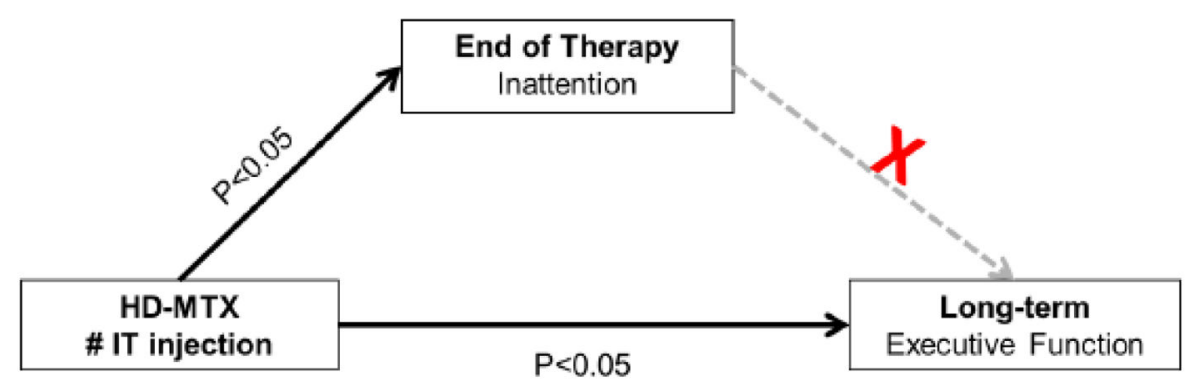

Figure 2b

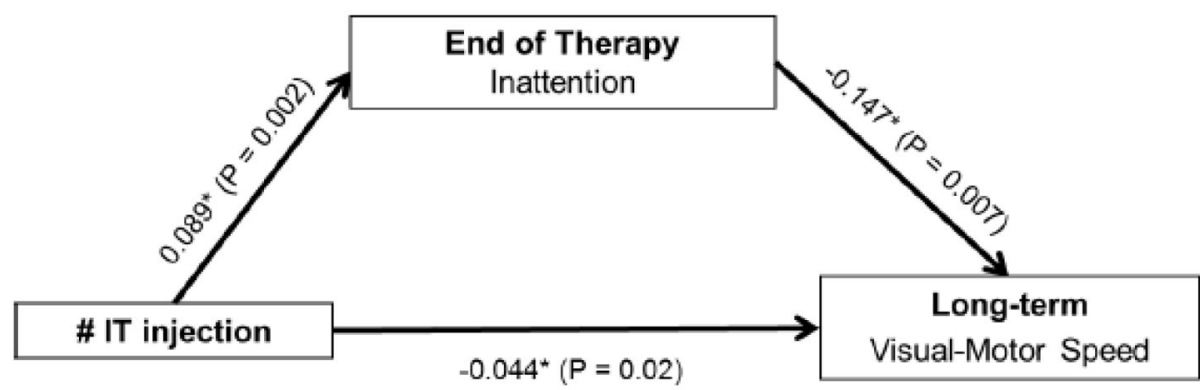

Figure 2.

Figure 2a. No Mediation effect of chemotherapy on executive function at long-term followup through behavior inattention at the end of therapy.

Figure 2b. Mediation effect of intrathecal chemotherapy on processing speed at long-term follow-up through parent-reported behavioral inattention at the end of therapy. Direct effect: $\beta=-0.044, p=0.02 ;$ Indirect effect: $\beta=(0.089) \times(-0.0147)=-0.013, p=0.04$ 


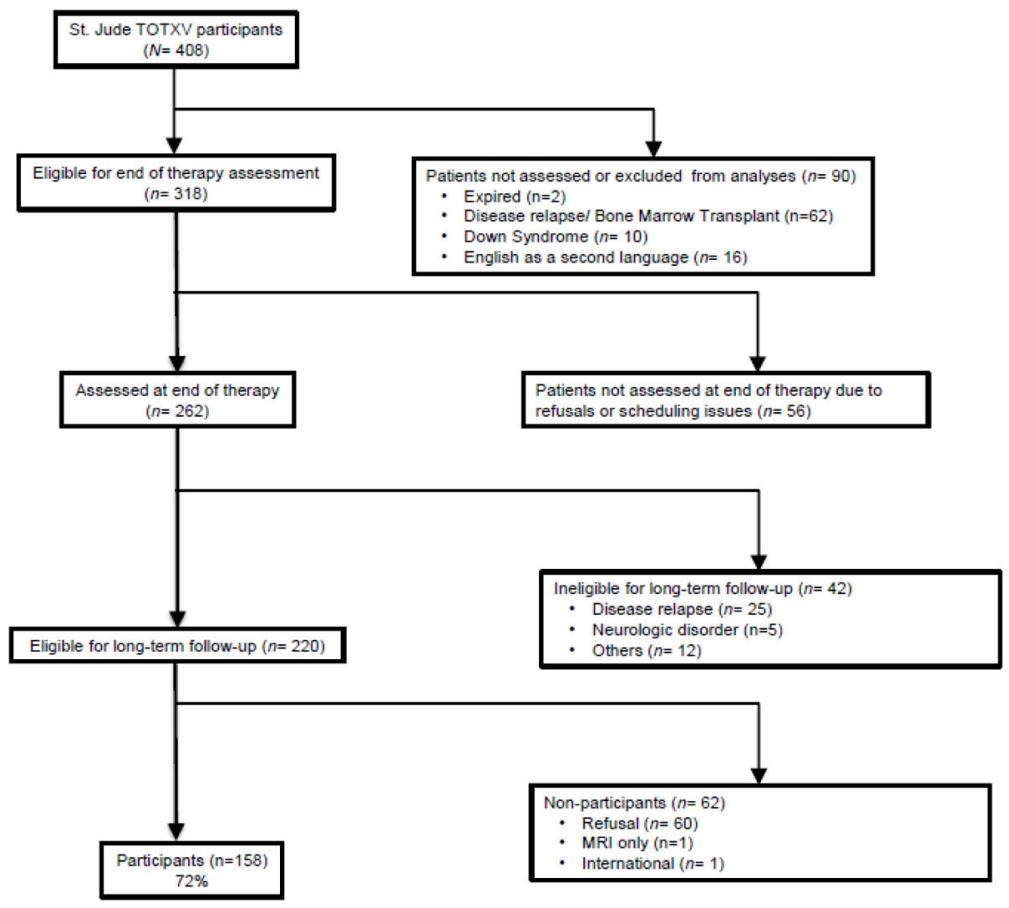

\section{Consort.}

Consort diagram of participants in longitudinal analysis. Of the 318 survivors eligible for end of therapy assessment, 262 (82\%) participated. 220 of these 262 were eligible for longterm follow-up and 158 (72\%) participated. 


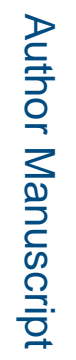

록

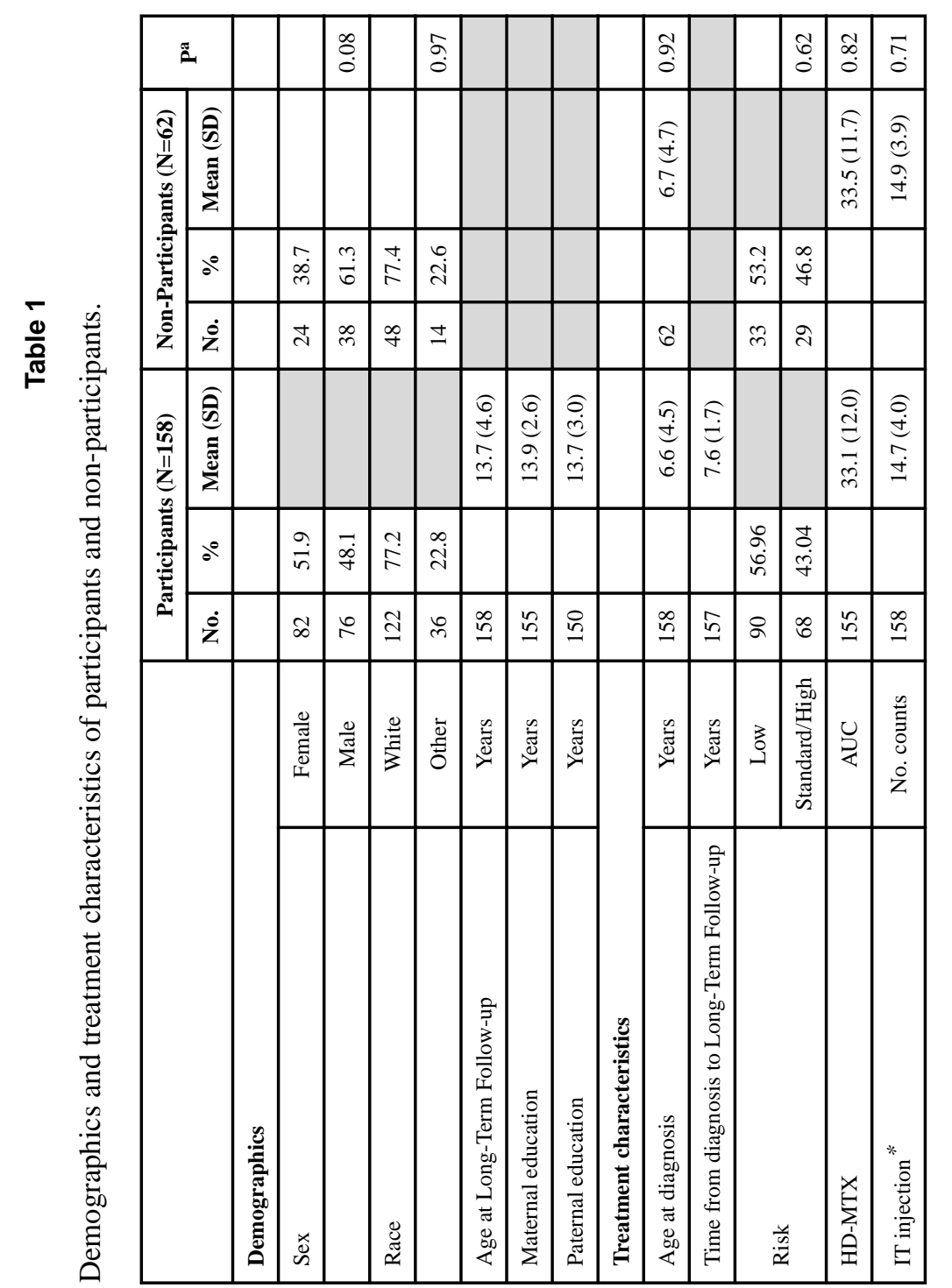

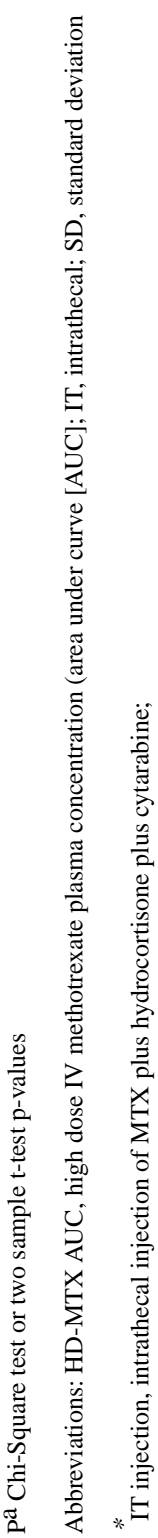

I

J Cancer Surviv. Author manuscript; available in PMC 2019 June 01. 


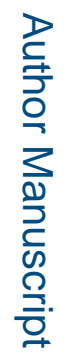

\begin{tabular}{|c|c|c|c|c|c|c|c|c|c|c|c|c|c|c|c|c|c|c|c|c|c|}
\hline$\hat{z}$ & 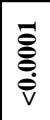 & 寺 & $\begin{array}{l}\overline{\bar{z}} \\
\stackrel{\bar{v}}{\mathrm{v}}\end{array}$ & 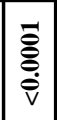 & 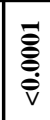 & 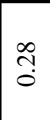 & & 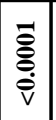 & $\mid \begin{array}{l}n \\
0 \\
0 \\
0\end{array}$ & 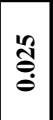 & 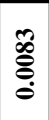 & $\overline{\text { ș }}$ & $\mid \begin{array}{c}\hat{a} \\
\dot{0} \\
\dot{0}\end{array}$ & 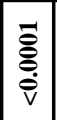 & $\begin{array}{l}\text { ôे } \\
\stackrel{0}{0} \\
\stackrel{0}{0}\end{array}$ & $\stackrel{\Xi}{0}$ & $\begin{array}{l}\overrightarrow{\bar{t}} \\
\stackrel{\bar{v}}{\mathrm{v}}\end{array}$ & $\begin{array}{l}0 \\
0 \\
0\end{array}$ & $\hat{m}$ & $\begin{array}{l}\infty \\
\stackrel{0}{0} \\
\stackrel{0}{0}\end{array}$ & 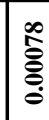 \\
\hline 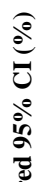 & $\mid \begin{array}{c}n \\
n \\
\infty \\
1 \\
r \\
\infty \\
\infty\end{array}$ & $\mid \begin{array}{c}0 \\
\infty \\
1 \\
1 \\
m\end{array}$ & $\begin{array}{c}\vec{a} \\
\vec{g} \\
1 \\
\vec{a}\end{array}$ & $\begin{array}{c}-\overrightarrow{0} \\
\dot{\infty} \\
1 \\
1 \\
\infty \\
\infty \\
\infty\end{array}$ & $\begin{array}{l}= \\
\tilde{\delta} \\
1 \\
m \\
m\end{array}$ & $\begin{array}{c}0 \\
\infty \\
0 \\
1 \\
0 \\
\dot{0}\end{array}$ & 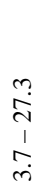 & $\begin{array}{l}0 \\
\dot{2} \\
1 \\
\tilde{n} \\
\end{array} \mid$ & $\mid \begin{array}{l}\hat{i} \\
\hat{i} \\
1 \\
\tilde{o}\end{array}$ & $\begin{array}{c}2 \\
\vec{\lambda} \\
1 \\
r\end{array}$ & $\begin{array}{c}0 \\
\vec{j} \\
1 \\
1 \\
\underline{m}\end{array}$ & $\begin{array}{l}n \\
\infty \\
0 \\
1 \\
n \\
n\end{array}$ & $\mid \begin{array}{c}\vec{r} \\
\vec{i} \\
1 \\
\tilde{m} \\
0\end{array}$ & $\mid \begin{array}{c}r \\
\dot{m} \\
1 \\
r \\
r\end{array}$ & $\begin{array}{l}\stackrel{i}{\vec{\lambda}} \\
1 \\
r \\
f\end{array}$ & $\begin{array}{c}\infty \\
0 \\
2 \\
1 \\
n \\
n\end{array}$ & $\begin{array}{l}0 \\
\dot{0} \\
1 \\
\dot{1} \\
\dot{H}\end{array}$ & $\begin{array}{l}\overrightarrow{0} \\
\overrightarrow{2} \\
1 \\
0\end{array}$ & $\begin{array}{c}\hat{a} \\
0 \\
1 \\
n \\
0\end{array}$ & $\begin{array}{l}0 \\
\vec{i} \\
1 \\
-1\end{array}$ & $\begin{array}{l}0 \\
\text { in } \\
1 \\
0 \\
0 \\
\text { in }\end{array}$ \\
\hline
\end{tabular}

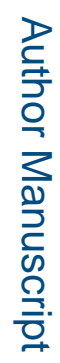

를

鯶

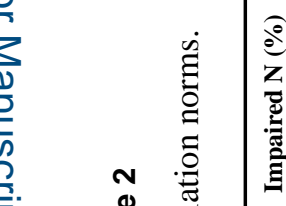

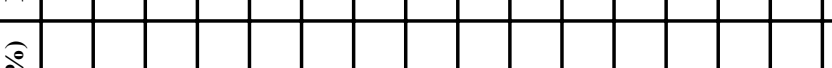

元

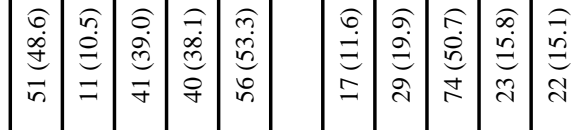

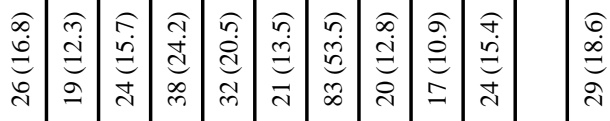

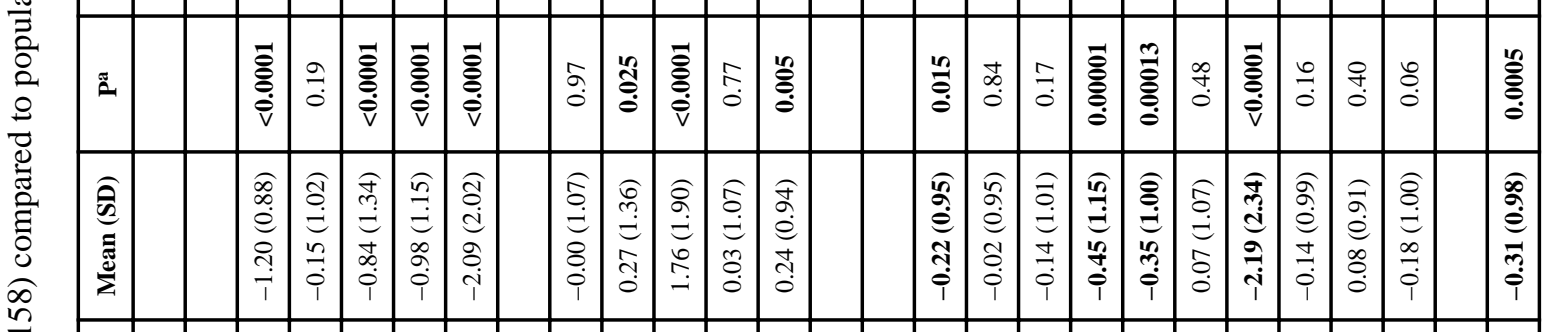

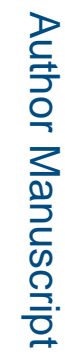

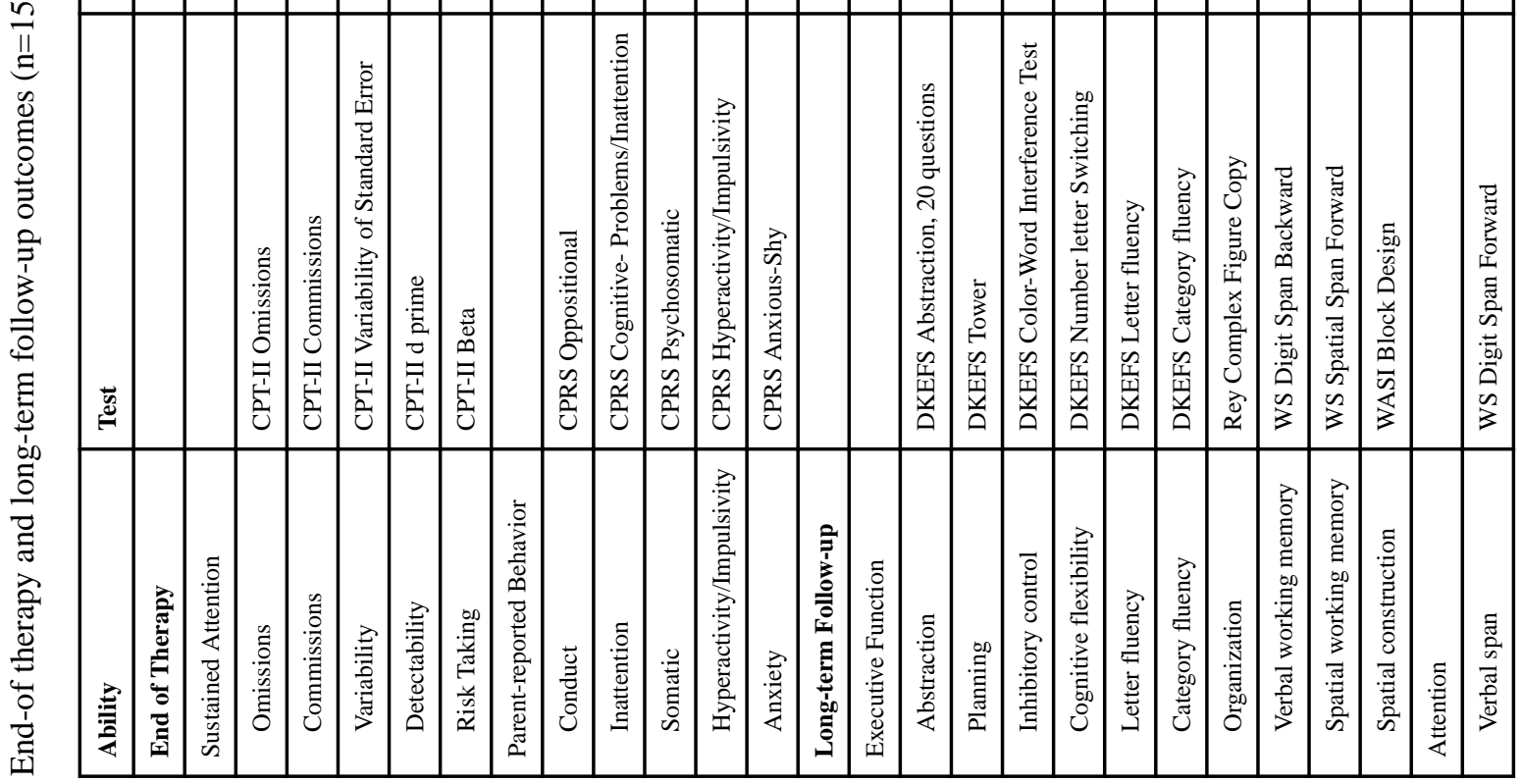

J Cancer Surviv. Author manuscript; available in PMC 2019 June 01. 
로을

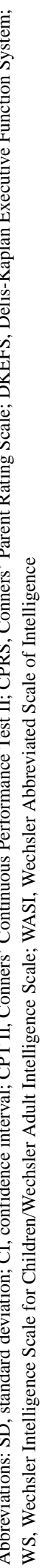

J Cancer Surviv. Author manuscript; available in PMC 2019 June 01. 


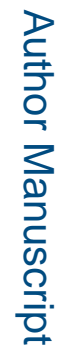

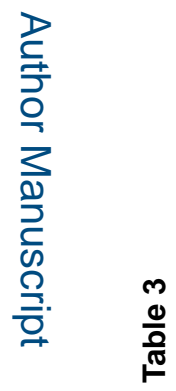

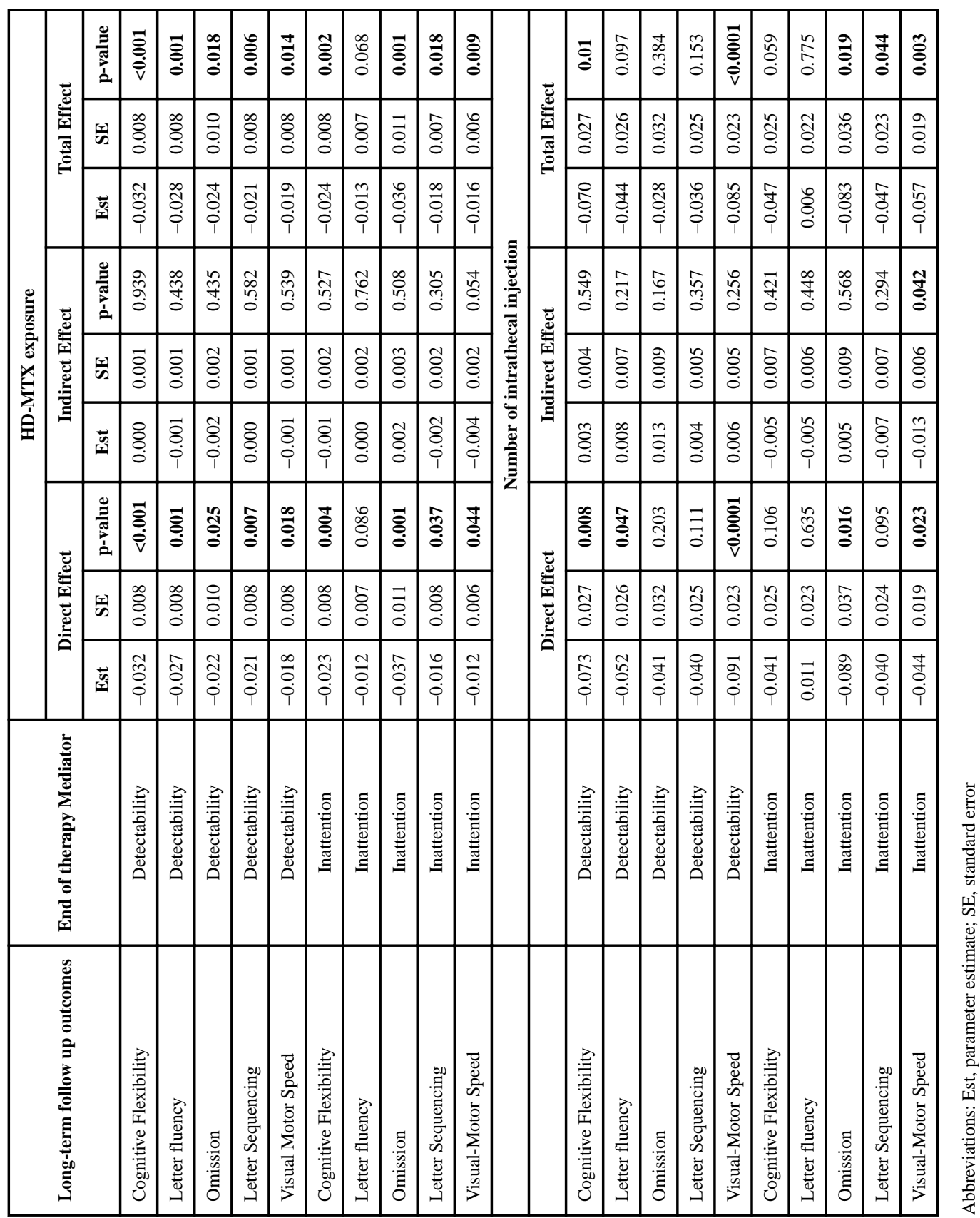

J Cancer Surviv. Author manuscript; available in PMC 2019 June 01. 